\title{
On a Class of Distributions Defined by the Relationship Between Their Density and Distribution Functions
}

\section{M.C. JONES}

Department of Statistics, The Open University, Walton Hall, Milton Keynes, MK7 6AA, U.K.

Knowledge concerning the family of univariate continuous distributions with density function $f$ and distribution function $F$ defined through the relation $f(x)=F^{\alpha}(x)(1-F(x))^{\beta}, \alpha, \beta \in \mathcal{R}$, is reviewed and modestly extended. Symmetry, modality, tail behaviour, order statistics, shape properties based on the mode, L-moments and - for the first time - transformations between members of the family are the general properties considered. Fully tractable special cases include all the complementary beta distributions (including uniform, power law and cosine distributions), the logistic, exponential and Pareto distributions, the Student $t$ distribution on 2 degrees of freedom and, newly, the distribution corresponding to $\alpha=\beta=5 / 2$. The logistic distribution is central to some of the developments of the article.

Keywords Complementary beta distribution; Logistic distribution; Quantile density function.

Mathematics subject classification Primary 62E10; Secondary 60E05. 


\section{Introduction}

Let $F$ and $f=F^{\prime}$ denote the probability distribution and density functions of a univariate continuous distribution. Define a class of distributions through the functional equation

$$
f(x)=f(x ; \alpha, \beta)=F^{\alpha}(x)(1-F(x))^{\beta}
$$

where $\alpha$ and $\beta$ are real-valued shape parameters. Expression (1) has arisen somewhat haphazardly in the literature over the years, so my aim in this article is to collect and modestly extend available information on this class of distributions; it is not especially my purpose either to commend these distributions to the reader or to denigrate them. As usual in distribution theoretical work, I need deal mostly only with canonical forms of densities ignoring location and scale parameters, although they would be of prime importance in fitting such distributions to data. However, as noted by Kent (2004), the constant of proportionality in (1) can be taken to be unity because any non-unit constant could be absorbed into the scale parameter. (Actually, I sometimes play fast and loose with the constant of proportionality.) The special case of (1) in which $\alpha=\beta=1$ is probably most familiar to readers: the distribution satisfying (1) is then the logistic distribution (e.g. Balakrishnan, 1992), a distribution that will play a central role in this paper.

Let $Q=F^{-1}$ and $q=Q^{\prime}$ denote the quantile and quantile density functions associated with $F$. An equivalent form to (1) is

$$
q(u)=u^{-\alpha}(1-u)^{-\beta}, \quad 0<u<1 .
$$

Formulation (2) makes it clear that there is a unique distribution associated with each combination of $\alpha$ and $\beta$ and it is in form (2) that much of the work on these distributions has taken place. Kamps (1991) is an important reference.

The remainder of the article is in two sections. A (not altogether successful) attempt has been made to separate the work into known and/or relatively immediate properties and special cases in Section 2 and novel properties and 
special cases in Section 3. The latter properties include (Section 3.2) work on transformations between members of family (1) which is probably the main novel contribution of this article. In considering special cases, I am particularly keen to concentrate on cases that I term 'fully tractable' in as much as each of their density, distribution and quantile functions is available in closed form, in addition to their quantile density functions. The main novel fully tractable special case turns out to correspond to $\alpha=\beta=5 / 2$ in (1)!

\section{Simple and Known Properties and Special Cases of Distribution}

\subsection{General Properties}

The special cases of distribution (1) that are symmetric (about zero) will be of particular interest in what follows and they correspond precisely to the cases where $\alpha=\beta$.

The derivative of density (1) is

$$
f^{\prime}(x)=\frac{f^{2}(x)}{F(x)(1-F(x))}\{\alpha-(\alpha+\beta) F(x)\} .
$$

If a mode or antimode $x_{0}$ exists it satisfies $F\left(x_{0}\right)=\alpha /(\alpha+\beta)$ at which point $f^{\prime \prime}\left(x_{0}\right)=-K_{\alpha \beta}(\alpha+\beta)$ for $K_{\alpha \beta}>0$. The distributions are therefore unimodal if $\alpha, \beta>0$, uniantimodal if $\alpha, \beta<0$, monotone nondecreasing if $\alpha \leq 0, \beta \geq 0$ and monotone nonincreasing if $\alpha \geq 0, \beta \leq 0$.

The principal role of the shape parameters is to control the tail weights of the distribution; in fact, $\alpha$ controls the left-hand tail, $\beta$ the right. Let us concentrate on $\beta$ and the right-hand tail, obvious analogous results holding for $\alpha$ and the left-hand tail or for both tails when $\alpha=\beta$. Consideration of formulation (2) in particular leads swiftly, via a little calculus, to the following:

(i) when $\beta<1, f(x) \sim(1-x)^{\beta /(1-\beta)}$ as $x \rightarrow 1$.

(ii) when $\beta=1, f(x) \sim e^{-x}$ as $x \rightarrow \infty$;

(iii) when $\beta>1, f(x) \sim x^{-\beta /(\beta-1)}$ as $x \rightarrow \infty$; 
Thus, there is a natural progression through distributions with a finite boundary when $\beta<1$, via an exponentially decaying tail when $\beta=1$, to heavy, power, tails, when $\beta>1$. This tail behaviour, at least for $\beta \geq 1$, has been mentioned by Kent (2004) and a subset thereof by Akhundhov, Balakrishnan and Nevzorov (2004). A consequence is that $r$ th moments exist provided $r<1 /(\max (\alpha, \beta)-1)$. In the symmetric case, a nice analogy is with the classical Pearson family of distributions which progresses through beta distributions on finite support, via the normal rather than logistic distribution, to Student $t$ distributions, $t_{\nu}$, with density tails going as $|x|^{-(\nu+1)}$ where $\nu$ is the degrees of freedom. It might be helpful to equate $\beta>1$ with $1+\nu^{-1}$, i.e. $\nu=1 /(\beta-1)$, else note that the whole range of tail behaviours from exponential $(\nu=\infty)$ to Cauchy-like $(\nu=1)$ corresponds to $1 \leq \beta \leq 2$.

Using the standard formula (David and Nagaraja, 2003, p.10) for the density $g$, say, associated with $X_{i: n}$, the $i$ th order statistic of a random sample of size $n$ from the distribution $F$, it is immediate that

$$
g(x)=\{B(i, n+1-i)\}^{-1} F^{i+\alpha-1}(x)(1-F(x))^{n+\beta-i}
$$

where $B(\cdot, \cdot)$ is the beta function. This affords a number of simplifications in dealing with expectations of functions of order statistics from distribution $F$, for which see Kamps (1991), Kamps and Mattner (1993) and Jones (2002a, Section 2.6).

\subsection{Special Cases}

Kamps (1991) and Jones (2002a) are the principal sources of existing special cases of distribution (1). Kamps restricted attention to integer $\alpha$ and $\beta$; Jones's main innovations referred to (real and integer) $\alpha$ and $\beta$ less than 1 .

2.2.1. $\alpha, \beta<1$. The entire set of distributions (1) for which both $\alpha$ and $\beta$ are less than 1 and hence $F$ has finite support have been described by Jones (2002a). I called them complementary beta distributions because their distribution function is the quantile function of the beta distribution and vice versa (likewise for density and quantile density functions). Setting $\alpha=1-a$, $\beta=1-b$ where $a$ and $b$ are the parameters used in Jones (2002a), the density 
of the complementary beta distribution on $(0,1)$ turns out to be

$$
f_{C}(u)=B(1-\alpha, 1-\beta)\left\{\mathcal{I}_{u}(1-\alpha, 1-\beta)\right\}^{\alpha}\left\{1-\mathcal{I}_{u}(1-\alpha, 1-\beta)\right\}^{\beta}
$$

where $\mathcal{I}_{u}(1-\alpha, 1-\beta)$ is the inverse of the incomplete beta function ratio, i.e. the quantile function of the usual beta distribution on $(0,1)$, with parameters $1-\alpha, 1-\beta$ or, equivalently, the distribution function of the complementary beta distribution. (Note that this is actually a case where, to keep support on $(0,1)$, a non-unit constant of proportionality has been taken.)

Special cases of the complementary beta distribution include the uniform distribution when $\alpha=\beta=0$, power law distributions with density proportional to $u^{\alpha /(1-\alpha)}$ when $\beta=0$ and with density proportional to $(1-u)^{\beta /(1-\beta)}$ when $\alpha=0$, the sine or cosine distribution when $\alpha=\beta=1 / 2$ and a further tractable special case when $\alpha=\beta=-1$. That the sine/cosine distribution satisfies (1) with $\alpha=\beta=1 / 2$ was also noted by Nevzorov, Balakrishnan and Ahsanullah (2003). See Jones (2002a) for much more on the complementary beta distribution.

2.2.2. $\alpha=\beta \geq 1$. The logistic distribution has already been mentioned as the case $\alpha=\beta=1$. Rather more surprising, perhaps, is that the distribution associated with $\alpha=\beta=3 / 2$ is the Student $t$ distribution on 2 degrees of freedom. This has been noted by Jones (2002a,b) and Nevzorov, Balakrishnan and Ahsanullah (2003). Things become less tractable for larger $\alpha=\beta$. For example, in the case $\alpha=\beta=2$, one can readily obtain the quantile function by integration of (2) to be

$$
Q_{2}(u)=2 \log \left(\frac{u}{1-u}\right)+\frac{2 u-1}{u(1-u)}
$$

but this is not explicitly invertible. Note that this is the symmetric member of family (1) with Cauchy-like heavy tails, its density behaving as $|x|^{-2}$ as $x \rightarrow \infty$.

2.2.3. $\alpha=0, \beta \geq 1$. The distributions in the case $\beta>1$ are Pareto distributions (Kamps, 1991). When $\beta=1$, the distribution is, of course, the 
exponential. In fact, the defining property in that case is constant (unit) hazard rate: $f(x)=1-F(x)$. Since the distributions with $\alpha=0$ and $\beta<1$ are power law distributions on finite support, I note in Jones (2002a) that all cases with $\alpha=0$ are covered by the generalized Pareto distribution (Hosking and Wallis, 1987). Distributions defined by taking $\beta=0$ and arbitrary $\alpha=\alpha_{1}$ are the distributions of $-X$ where $X$ follows the distribution with $\alpha=0$ and $\beta=\alpha_{1}$.

2.2.4. More cases without explicitly invertible quantile functions. When $\alpha$ and $\beta$ are integers, expressions for quantile functions associated with quantile density functions (2) are available by integration. Kamps (1991, cases (iv)-(ix)) provides general expressions that cover all these cases (including (4) which can be obtained from Kamps's case (ix)). However, these quantile functions are not explicitly invertible in general and hence are not fully tractable in the sense I am employing here.

The cases $1<\alpha<2$ and $1<\beta<2$ such that $\alpha+\beta=3$ arise along with their explicit but generally non-invertible quantile functions in Balakrishnan and Akhundhov (2003) and Akhundhov, Balakrishnan and Nevzorov (2004). These authors characterize the distributions by linearity of certain expectations given the value of an order statistic.

\section{Further Properties and Special Cases of Distribution (1)}

\subsection{Location, Scale, Skewness and Kurtosis}

Two approaches to scalar summaries of location, scale and shape yield (mostly) simple results for distributions (1). First, these distributions are particularly amenable to scalar summaries of location, scale and shape based on the mode, $x_{0}=F^{-1}(\alpha /(\alpha+\beta))$, when $\alpha, \beta>0$. The mode itself describes location. The reciprocal of the value of the density at its mode is

$$
\frac{1}{f\left(x_{0}\right)}=\frac{(\alpha+\beta)^{\alpha+\beta}}{\alpha^{\alpha} \beta^{\beta}}
$$

and this is a mode-based measure of the spread of the distribution. It can also 
be immediately noted that the mode is situated at the $\alpha /(\alpha+\beta)$ 'th quantile of the distribution. This can be formalised into a measure of skewness via Arnold and Groeneveld's (1995) formula $\gamma=1-2 F\left(x_{0}\right)$, which takes the simple value

$$
\gamma=\frac{\beta-\alpha}{\alpha+\beta}
$$

in this case. This is, of course, an increasing function of $\beta$ for fixed $\alpha$ and a decreasing function of $\alpha$ for fixed $\beta$. Critchley and Jones (2006, Section 5) have argued for a corresponding measure of kurtosis which, in the symmetric case where $\alpha=\beta$ for simplicity, takes the value $\delta=2\left\{f(\pi) / f\left(x_{0}\right)\right\}-1$ where $\pi$ is either point of inflection of the density, assuming such exists. It turns out that what is needed is that $\beta>1 / 2$ so that $F(\pi)=\left(1+(4 \beta-1)^{-1 / 2}\right) / 2$ and

$$
\delta=2\left\{\frac{2(2 \beta-1)}{4 \beta-1}\right\}^{\beta}-1,
$$

which appears to be an increasing function of $\beta>1 / 2$.

Second, L-moments (Hosking, 1990) exist provided the mean of the distribution exists i.e. for $\alpha, \beta<2$. Jones (2002a, Section 2.7) gave a general formula for second and higher L-moments, $\lambda_{r}$, which I now observe applies equally well for larger $\alpha, \beta$ than the $\alpha, \beta<1$ restriction - to complementary beta distributions - made there: set $a=1-\alpha, b=1-\beta$ in (4) of Jones (2002a) and remove the divisor $B(a, b)$ to correspond to unit constant of proportionality in (1). A general formula for the mean (Jones, 2002a, Section 2.6) pertains for $\alpha, \beta<1$ but not for larger values of the parameters. The second L-moment, one-half of Gini's mean difference, is $B(2-\alpha, 2-\beta)$. More interesting here, but really only transcribed from Jones (2002a, (5)), are the third and fourth L-moment ratio measures of skewness $\left(\tau_{3}\right)$ and kurtosis $\left(\tau_{4}\right)$. The former is

$$
\tau_{3}=\frac{\beta-\alpha}{4-\alpha-\beta}
$$

also an increasing function of $\beta$ for fixed $\alpha$ and a decreasing function of $\alpha$ for fixed $\beta$. The latter, again in the symmetric case for comparative purposes, 
is

$$
\tau_{4}=\frac{\beta}{2(5-2 \beta)},
$$

a simpler and certainly increasing function of $\beta<2$.

\subsection{Transformation Properties}

Concentrate initially on symmetric cases, $\alpha=\beta$. Set $f_{\alpha}(x)=f(x ; \alpha, \alpha)$ and let $X_{\alpha} \sim f_{\alpha}$ where $\sim$ means 'is distributed as' and what follows the $\sim$ sign might be either a density or another random variable.

Theorem 1. For $\alpha \neq 0$,

$$
X_{\beta} \sim T\left(X_{\alpha}\right) \quad \text { where } \quad T^{\prime}(x)=f_{\alpha}^{1-(\beta / \alpha)}(x) .
$$

Proof: By standard transformation of variables,

$$
\begin{aligned}
f_{\beta}\left(x_{\beta}\right) & =\frac{f_{\alpha}\left(T^{-1}\left(x_{\beta}\right)\right)}{T^{\prime}\left(T^{-1}\left(x_{\beta}\right)\right)}=\left\{F_{\beta}\left(x_{\beta}\right)\left(1-F_{\beta}\left(x_{\beta}\right)\right)\right\}^{\beta} \\
& =\left\{F_{\alpha}\left(T^{-1}\left(x_{\beta}\right)\right)\left(1-F_{\alpha}\left(T^{-1}\left(x_{\beta}\right)\right)\right)\right\}^{\beta}=f_{\alpha}^{\beta / \alpha}\left(T^{-1}\left(x_{\beta}\right)\right)
\end{aligned}
$$

and the result follows from the second and final expressions in the chain of equalities.

It is particularly tempting to relate other symmetric members of family (1) to the logistic distribution by transformation. A logistic random variable is denoted $X_{1}$.

Corollary 1.

$$
X_{\beta} \sim T\left(X_{1}\right) \quad \text { where } \quad T^{\prime}(x)=\frac{\left(1+e^{x}\right)^{2(\beta-1)}}{e^{(\beta-1) x}}=\{2 \cosh (x / 2)\}^{2(\beta-1)} .
$$

It is interesting to note that in this case $f_{\beta}(x)=\exp \left(\beta T^{-1}(x)\right) /$ $\left(1+\exp \left(T^{-1}(x)\right)^{2 \beta}\right.$, the form - sans normalisation constant - of a symmetric $\log F$ density (e.g. Jones, 2006a) at $T^{-1}(x)$. 
Further explicit transformations need to be computed on a case-by-case basis. A couple of known transformations of the logistic that are reproduced by (6) are: (a) $T(x)=e^{x} /\left(1+e^{x}\right)$, the probability integral transform for the logistic leading to the uniform distribution, $\beta=0$; and (b) $T(x)=$ $4 \sinh (x / 2)$, the simple transformation of the logistic to the $t_{2}$ distribution (Jones, 2004, 2006b) corresponding to $\beta=3 / 2$. Further work using (6) will follow in Section 3.3.

There is also an extension of Theorem 1 to transformations from symmetric $F$ 's to asymmetric $F$ 's; it is given next. (I do not have as general a result for transformations from asymmetric starting points although some special cases are straightforward.) Of course, (7) to follow reduces to (5) when $\gamma=\beta$, as will (8) to (6).

Theorem 2. Let $X_{\gamma \beta} \sim f(\cdot ; \gamma, \beta)$. For $\alpha \neq 0, X_{\gamma \beta} \sim T\left(X_{\alpha}\right)$ where

$$
T^{\prime}(x)=\frac{2^{\gamma+\beta} f_{\alpha}(x)}{\left(1+\sqrt{1-4 f_{\alpha}^{1 / \alpha}}\right)^{\gamma}\left(1-\sqrt{1-4 f_{\alpha}^{1 / \alpha}}\right)^{\beta}} .
$$

Proof: Note that $F_{\alpha}(x)=(1 / 2)\left(1+\sqrt{1-4 f_{\alpha}^{1 / \alpha}}\right)$ (and that $\left.f_{\alpha}^{1 / \alpha} \leq 1 / 4\right)$ and proceed similarly to the proof of Theorem 1 .

Corollary 2.

$$
X_{\gamma \beta} \sim T\left(X_{1}\right) \text { where } T^{\prime}(x)=\frac{\left(1+e^{x}\right)^{\gamma+\beta-2}}{e^{(\beta-1) x}}=\left(1+e^{x}\right)^{\gamma-1}\left(1+e^{-x}\right)^{\beta-1} .
$$

A simple but not explicitly invertible consequence of (8) is the transformation to the distribution with $\gamma=2, \beta=1: T_{12}(x)=x+e^{x}$.

The transformation can be more generally fully specified for the complementary beta distribution as next.

Corollary 3. Let $\gamma, \beta<1$. Then

$$
X_{\gamma \beta} \sim T\left(X_{1}\right) \quad \text { where } \quad T(x)=B_{e^{x} /\left(1+e^{x}\right)}(1-\gamma, 1-\beta)
$$


and $B_{y}(\cdot, \cdot)$ is the incomplete beta function at $y$.

Note that the versions of the complementary beta distribution followed by $X_{\gamma \beta}$ are actually on support $(1-\gamma, 1-\beta)$. Observation (a) between Corollary 1 and Theorem 2 makes Corollary 3 rather immediate: its transformation is composed of the transformation to uniformity followed by the transformation $B_{U}(1-\gamma, 1-\beta)$ (Jones, 2002) to the complementary beta distribution.

\section{3. $\alpha=\beta=5 / 2$ and Similar Special Cases}

Consider again the transformations from the logistic to other symmetric $F$ distributions given by the second formula in (6), now subscripted by $\beta$ : $T_{\beta}^{\prime}(x)=\{2 \cosh (x / 2)\}^{2(\beta-1)}$. Explicit formulae for indefinite integrals of some powers of cosh are given in Gradshteyn and Ryzhik (1994, Sections 2.414 and 2.423): these afford explicit formulae for $T_{\beta}(x)$ for a number of integer and half-integer positive and negative values of $\beta$. For example, when $\beta=2, T_{2}(x)=2(\sinh (x)+x)$. If $Q_{1}(u)=\log (u /(1-u))$ is the logistic quantile function, then application of this transformation - via $T_{2}\left(Q_{1}(u)\right)$ - does indeed lead to the quantile function (4) associated with $\beta=2$.

Continuing with $\beta>1$, half-integer values are most tractable. With $\beta=$ $3 / 2$ understood, next is $\beta=5 / 2$. For this case, $T_{5 / 2}(x)=16 \sinh (x / 2)(1+$ $\left.(1 / 3) \sinh ^{2}(x / 2)\right)$ and $T_{5 / 2}^{-1}(x)=2 \sinh ^{-1}(s)$ where $s=m^{1 / 3}-m^{-1 / 3}$ and

$$
m=\frac{3}{32} x+\sqrt{\frac{9}{1024} x^{2}+1}
$$

It follows that

$$
Q_{5 / 2}(u)=\frac{2(2 u-1)\left(8 u-8 u^{2}+1\right)}{3 u^{3 / 2}(1-u)^{3 / 2}}
$$

and that this is explicitly invertible to give

$$
F_{5 / 2}(x)=\frac{1}{2}\left(1+\frac{m^{1 / 3}-m^{-1 / 3}}{\sqrt{m^{2 / 3}+m^{-2 / 3}-1}}\right)
$$

so that

$$
f_{5 / 2}(x)=\left(\frac{1}{2 \sqrt{m^{2 / 3}+m^{-2 / 3}-1}}\right)^{5} .
$$


So, this is a novel member of family (1) whose density, distribution, quantile and, necessarily, quantile density functions can all be provided explicitly. However, (9) is extremely heavy-tailed. It is plotted against the equally heavy-tailed $t_{2 / 3}$ distribution in Figure 1, having first been rescaled to match the value of the latter's density at zero. The two densities are, clearly, very similar to one another.

\section{$* * * \quad$ Figure 1 about here $* * *$}

The transformations of the logistic distribution leading to the sine or cosine distribution $(\beta=1 / 2)$ and the "further tractable special case when $\alpha=\beta=-1$ " of Jones (2002a) are $T_{1 / 2}(x)=\tan ^{-1}(\sinh (x / 2))$ and $T_{-1}(x)=$ $\tanh (x / 2)\left\{3-\tanh ^{2}(x / 2)\right\} / 24$, respectively.

\section{References}

Akhundhov, I.S., Balakrishnan, N., Nevzorov, V.B. (2004). New characterizations by properties of midrange and related statistics. Commun. Statist. - Theory Meth. 33:3133-3143.

Arnold, B.C., Groeneveld, R.A. (1995). Measuring skewness with respect to the mode. Amer. Statist. 49:34-38.

Balakrishnan, N. (ed.) (1992). Handbook of the Logistic Distribution. New York: Dekker.

Balakrishnan, N., Akhundhov, I.S. (2003). A characterization by linearity of the regression function based on order statistics. Statist. Probab. Lett. $63: 435-440$.

Critchley, F., Jones, M.C. (2006). Asymmetry and gradient asymmetry functions: density-based skewness and kurtosis. Under consideration. An earlier version of this paper is Open University Department of Statistics Technical Report 05/06; see http://statistics.open.ac.uk/ TechnicalReports/TechnicalReportsIntro.htm

David, H.A., Nagaraja, H.N. (2003). Order Statistics. Third edition. New York: Wiley. 
Gradshteyn, I.S., Ryzhik, I.M. (1994). Table of Integrals, Series, and Products. Fifth edition. San Diego: Academic Press.

Hosking, J.R.M. (1990). L-moments: analysis and estimation of distributions using linear combinations of order statistics. J. Roy. Statist. Soc. Ser. B 52:105-124.

Hosking, J.R.M., Wallis J.R. (1987). Parameter and quantile estimation for the generalized Pareto distribution. Technometrics 29:339-349.

Jones, M.C. (2002a). The complementary beta distribution. J. Statist. Planning Inference 104:329-337.

Jones, M.C. (2002b). Student's simplest distribution. Statistician 51:41-49.

Jones, M.C. (2004). Families of distributions arising from distributions of order statistics (with discussion). Test 13:1-43.

Jones, M.C. (2006a). The logistic and the $\log F$ distribution. In: Balakrishnan, N. ed., Handbook of the Logistic Distribution, Second Edition, New York: Dekker, to appear. Open University Department of Statistics Technical Report 06/01; see http://statistics.open.ac.uk/ TechnicalReports/TechnicalReportsIntro.htm

Jones, M.C. (2006b). On a class of distributions with simple exponential tails. Under consideration. Open University Department of Statistics Technical Report 06/02; see http://statistics.open.ac.uk/TechnicalReports/ TechnicalReportsIntro.htm

Kamps, U. (1991). A general recurrence relation for moments of order statistics in a class of probability distributions and characterizations. Metrika 38:215-225.

Kamps, U., Mattner, L. (1993). An identity for expectations of functions of order statistics. Metrika 40:361-365.

Kent, J.T. (2004). Contribution to the discussion of "Families of Distributions Arising From Distributions of Order Statistics" by M.C. Jones. Test 13:27-29. 
Kotz, S., Kozubowski, T.J., Podgórski, K. (2001). The Laplace Distribution and Generalizations; A Revisit With Applications to Communications, Economics, Engineering, and Finance. Boston: Birkhauser.

Nevzorov, V.B., Balakrishnan, N., Ahsanullah, M. (2003). Simple characterizations of Student's t(2)-distribution. Statistician 52:395-400. 
Figure 1. Density (8) corresponding to $\alpha=\beta=5 / 2$ (solid line) and Student $t$ density on $2 / 3$ degrees of freedom (dashed line). The former has been scaled to match the latter's density value at zero.

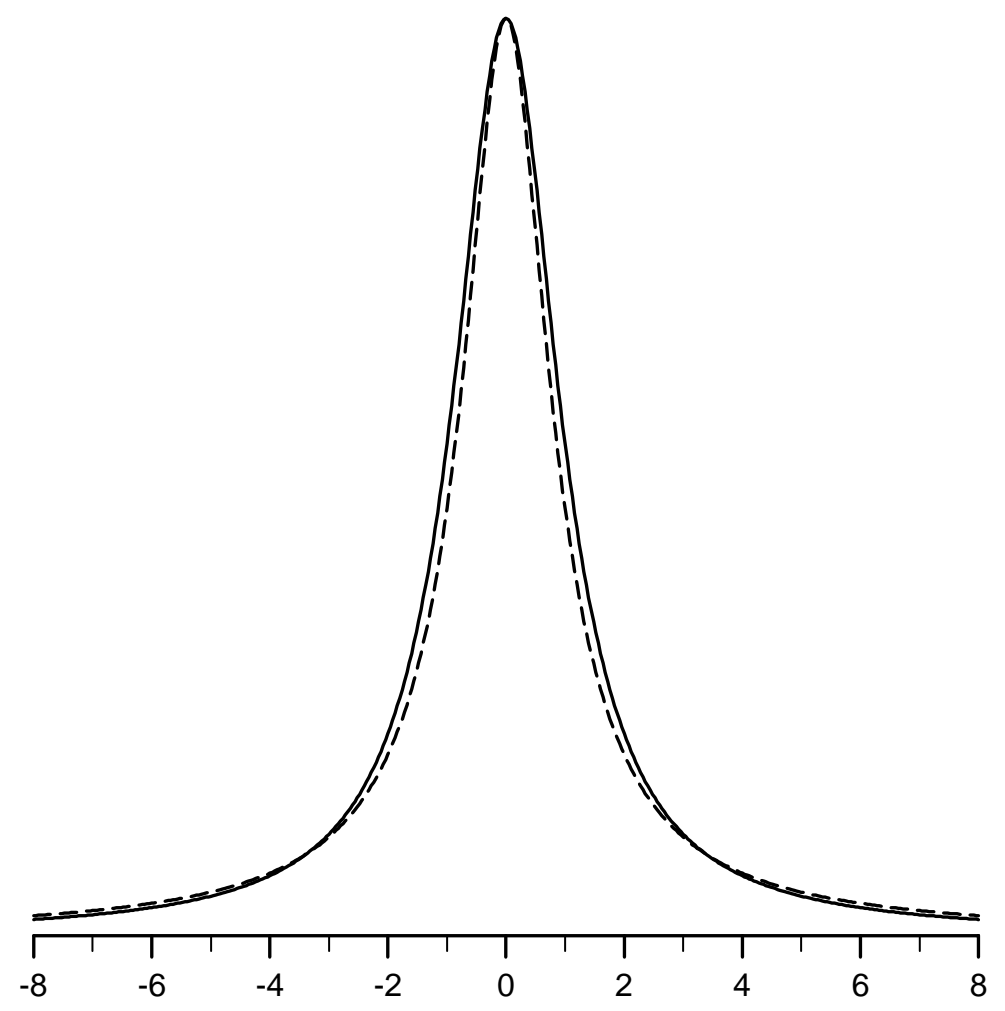

